\title{
René Guiart, Le feu sous la marmite
}

2001, Nouméa, Le Rocher-à-la-Voile, coll. « Documents pour servir à l'intelligence du temps présent » 5, $368 \mathrm{p}$.

Isabelle Leblic

\section{OpenEdition}

Journals

Édition électronique

URL : http://journals.openedition.org/jso/1238

DOI : $10.4000 /$ jso. 1238

ISSN : $1760-7256$

Éditeur

Société des océanistes

\section{Édition imprimée}

Date de publication : 1 juin 2003

Pagination : 118-119

ISSN : 0300-953x

\section{Référence électronique}

Isabelle Leblic, «René Guiart, Le feu sous la marmite », Journal de la Société des Océanistes [En ligne], 116 | Année 2003-1, mis en ligne le 26 mai 2008, consulté le 25 septembre 2020. URL : http:// journals.openedition.org/jso/1238; DOI : https://doi.org/10.4000/jso.1238 
du centre-ville dans les années 1984-1988, les attentats qui ravagèrent plusieurs établissements publics dont le palais de justice, les émeutes «blanches 》 de janvier 1985, les manifestations kanak au cœur de la ville, le couvre-feu, les coups de feu contre les Kanaks et même la mort d'un jeune manifestant kanak (Léopold Dawano)! Oubli des plus étonnants et décevants pour des tenants de l'histoire contemporaine ( $f f$. note 1) ; jadis c'est le bagne dont on ne parlait pas, aujourd'hui, ce sont les «événements ». Les auteurs auraient peutêtre dû arrêter leur anthologie au début des années 1980 et ne pas parler du tout de l'accord de Nouméa de 1998, d'autant plus que l'extrait (p. 117) qui en est donné renforce cette impression de partialité : «Des hommes et des femmes sont venus en grand nombre, aux $\mathrm{XIX}^{\mathrm{e}}$ et $\mathrm{Xx}^{\mathrm{e}}$ siècles, convaincus d'apporter le progrès, animés par leur foi religieuse, venus contre leur gré ou cherchant une nouvelle chance en NouvelleCalédonie. Ils se sont installés et y ont fait souche. Ils ont apporté avec eux leurs idéaux, leurs connaissances, leurs espoirs, leurs ambitions, leurs illusions et leurs contradictions ». Extrait des plus réducteurs ${ }^{2}$ s'il en est de cet accord ! Les auteurs, par leur oubli, veulentils dresser un tableau idyllique de Nouméa-lablanche ? Cela nuit à leur objectif d'écrire une anthologie de Nouméa donnant diverses représentations de cette ville au fil des années.

Les auteurs ont fait le choix d'une présentation chronologique depuis 1854 à nos jours, pourquoi pas ! Pour chacun de ces chapitres, ils ont donc retenu quelques dates qui sont illustrées par des extraits de textes et des illustrations (photos, cartes, plans, dessins...). Mais l'insertion de sous-chapitres thématiques et la multiplicité des témoignages introduisent certaines redondances (comme par exemple l'extrait de Jules Garnier p. 48 déjà cité p. 20). On peut aussi regretter que les sources ne soient pas mentionnées de façon claire sous chaque document ainsi présenté (on n'a qu'un auteur, les dates de publication - que l'on doit aller chercher en fin d'ouvrage - n'ont souvent rien à voir avec les repères chronologiques et on ne sait pas si ce sont des sources d'époque ou des documents postérieurs qui sont ainsi mélangés au fil des pages et mis sur un même plan). Aussi peut-on regretter que ne soient pas mentionnées les sources de chaque extrait cité et qu'il n'y ait pas d'analyse et de confrontation des différents documents traitant d'un même sujet, certains pouvant paraître à première vue contradictoire.
Le fait d'avoir mis côte à côte tous ces documents représente cependant un intérêt certain que la qualité des illustrations renforce.

\section{Isabelle LEBLIC,}

LACITO-CNRS

René Guiart, 2001, Le feu sous la marmite, Nouméa, Le Rocher-à-la-Voile, coll. « Documents pour servir à l'intelligence du temps présent » 5, $368 \mathrm{p}$.

Cet ouvrage imposant (368 p.) traite des revendications foncières en Nouvelle-Calédonie à travers la présentation presque au jour le jour du travail réalisé par le comité des terres de la côte Ouest auquel l'auteur a participé. En particulier, celui-ci nous renseigne sur une partie de l'histoire des revendications kanak antérieures aux fameux événements débutés en novembre 1984, donnant un éclairage sur une partie de l'histoire des revendications kanak souvent mal connue car n'ayant pas donné lieu à un développement médiatique en dehors du territoire de Nouvelle-Calédonie. Ces années avant 1984 sont pourtant cruciales dans la montée des revendications indépendantistes et ce témoignage nous éclaire donc sur des points souvent méconnus de cette histoire.

Après un avertissement de Jean Guiart (pp. 7-11), père de l'auteur et responsable de la collection, l'ouvrage est construit en huit chapitres intitulés: «Renaissance»; «Le retour au pays »; «Injustices »; « Un combat »; « La prison »; « L'escalade »; «La révolte »; «L'enlisement»; une courte bibliographie termine l'ensemble.

Le récit des aventures calédoniennes de René Guiart commence en mai 1980 et s'achève en décembre 1987. À ce titre, on peut dire qu'au fil des pages - écrites au départ sur place à Oundjo et reprises semble-t-il après -, on apprend beaucoup sur la façon concrète dont se sont passées nombre d'actions dont on n'avait entendu parler que sporadiquement. Les propos peuvent paraître parfois un peu naîfs, mais il n'en demeure pas moins que l'auteur a fait preuve d'un certain courage en s'engageant comme il l'a fait, même si-comme il le dit lui-même - il s'est trouvé pris dans ce mouvement un peu malgré lui. Comme nombre de personnes s'intéressant à la Nouvelle-Calédonie, il a été « saisi d'un sentiment de honte et de colère » (p. 33) en réali-

2. D'autres auteurs en retiennent des éléments de tout autre nature, comme par exemple : «Après avoir été soulagé par la signature de l'accord de Nouméa puis, dégrisé, s'être attaqué à la lecture dudit accord, tout un chacun, s'il est non kanak, n'a pu s'empêcher d'avoir l'une de ces deux réflexions : «Je n'y comprends rien » ou « On a fait trop de concessions aux Kanak ». Examinons ces concessions. La reconnaissance du fait colonial dans le préambule ? La restriction du corps électoral, fondement d'une nouvelle citoyenneté ? L'irréversibilité du transfert des compétences de l'État vers le territoire ? Et si nous examinions les concessions arrachées aux Kanak depuis la prise de possession de la Calédonie ? [...] Les concessions faites restent largement recevables au regard de ce qu'on y gagne : la paix, la poursuite du développement économique avec toujours l'aide de la France, et puis notre petit confort, notre travail, nos deux voitures, nos voyages de vacances, notre index (?) ... Et puis si j'avais été Kanak, j'aurais exigé encore plus de concessions... Et puis, que les non-Kanak arrêtent de faire [d'être] les gâtés de l'histoire! Les non-Kanak ne sont-ils pas les vrais vainqueurs de cette longue période de vingt-cinq ans qui vient de s'écouler et qui a vu le Peuple Kanak et ses amis reporter régulièrement et toujours à plus loin cette indépendance pour laquelle lui et ses amis ont lutté ? » (Éric H. Douyère, 2000, L'avenir de la Nouvelle-Calédonie en question(s). Du rééquilibrage des idées (II), Nouméa, île de Lumière, coll. «Libres écrits », 113 p., p. 33-35). Voir également le magnifique ouvrage de Jean-Claude Bourdais Nouméa culpa (Nouméa, éd. Rhizome, 2002) qui présente une toute autre vision du Nouméa contemporain. 
sant ce que la colonisation avait fait subir aux Kanaks au nom de la France : " Tristesse d'avoir eu une part, même indirecte, au sort qui lui [le peuple kanak] avait été fait. » (p. 33).

Mais peut-être que l'implication directe de l'auteur de ces lignes dans ces événements ne le rend pas forcément toujours très objectif. Ainsi, ses jugements sévères sur les groupes de pression - l'Union progressiste mélanésienne (UPM) exceptée puisque c'est le parti qu'il ralliera finalement - et sur les leaders kanak peuvent laisser perplexe. René Guiart revient notamment sur le fait que Machoro et Nonnaro auraient été sacrifiés par les responsables de l'Union calédonienne (UC) - parti majoritaire au sein du FLNKS - afin de ne pas compromettre leurs négociations en cours avec Edgard Pisani, envoyé fin 1984 comme hautcommissaire en Nouvelle-Calédonie pour résoudre la crise initiée le 18 novembre 1984! Aujourd'hui, en dehors de Pisani lui-même - le dernier à pouvoir le faire car le seul à être toujours en vie -, probablement personne ne peut dire ce qui s'est vraiment passé. L'opposition supposée ici entre Éloi Machoro et JeanMarie Tjibaou ( $c f$. p. 271 notamment) n'est pas une idée nouvelle et a déjà été évoquée par d'autres auteurs, sans être démontrée.

Quiconque s'intéresse de près ou de loin à la Nouvelle-Calédonie ou aux luttes de libération lira ce livre avec beaucoup d'intérêt. On se laisse emporter par le récit. En cela, l'ouvrage est réussi. On ne peut que regretter les problèmes d'édition, récurrents dans cette collection et qui nuisent souvent à la bonne compréhension. Ainsi, par exemple, l'appareil de notes au demeurant important se trouve en fin de chapitre, ce qui ne facilite pas la lecture. Sans compter que nombre d'appels de note n'ont pas leur correspondant en fin de chapitre : très nombreuses erreurs dans la numérotation, notes introuvables ou sans appels correspondants dans le texte, etc. L'agencement même des notes traduit une mauvaise conception de l'ouvrage. Ainsi, dans le chapitre intitulé « La révolte », avant-dernier du livre, plusieurs notes nous apportent de précieuses informations qu'il aurait été utile de trouver en début d'ouvrage. Par exemple, la note 13 (p. 303) nous présente Éloi Machoro dont on parle depuis le début; la note 17 (p. 304) nous explique l'origine du mot kanak - il est temps - ; la note 24 (qui apparaît sous le nombre 20 p. 304 !) nous dit d'où vient le mot caldoche ; la note 28 (22 p. 304) nous explique l'origine de l'UC et des autres groupes de pression indépendantistes ; idem pour la note 23 (p. 305) pour le FLNKS ; la note 26 (p. 306) qui apporte des précisions sur l'assassinat des dix hommes de Hienghène aurait été mieux à sa place pages 260-265. Enfin, il faut attendre la page 271 pour le renvoi à la note 33 (p. 307) et avoir confirmation que ce que l'auteur nomme au fil des pages « le journal-tambour » est en fait Les Nouvelles calédoniennes... Seuls ceux qui connaissent le pays pouvaient le supposer. La note 38 (p. 308) nous dit qui est Fessard Wabéalo dont il est question depuis quasiment le début de l'ouvrage comme compagnon de lutte de l'auteur; de même, la note 47 (p. 309) nous présente enfin François Burck. Et il faut attendre la note 43 (p. 308) pour comprendre que le Front calédonien et l'extrême droite locale sont inféodés au RPCR !

L'ouvrage apparaît ainsi comme un patchwork sans véritable unité, soit qu'il ait été rédigé en plusieurs fois sans relecture de l'ensemble, soit que l'ordre des chapitres ait été modifié pour la publication. Les nombreuses fautes de frappe ou d'orthographe renforce cette impression d'édition bâclée. Dommage! Car malgré toutes ces imperfections, cet ouvrage, que l'on peut qualifier de « récit de vie et de lutte », est à lire quand on s'intéresse de près ou de loin à la Nouvelle-Calédonie et/ou aux luttes de libération nationales.

\section{Isabelle LEBLIC,} LACITO-CNRS

Isabelle Revol, 2001. Fleur d'igname, Nouméa, Éditions Catherine Ledru, $24 \mathrm{p}$.

S'il n'est pas courant de trouver dans nos colonnes des comptes rendus de livres pour enfants, c'est avec enthousiasme que je me suis proposée de le faire à la réception de cet ouvrage. Car la qualité et la justesse de Fleur d'igname justifient à plus d'un titre qu'on en parle dans cette rubrique. En effet, ce conte merveilleux de la naissance de l'igname, imaginé, écrit et joliment illustré par l'auteur, est un récit qui, bien que n'étant pas une véritable légende de tradition orale kanak, rend à merveille toute l'atmosphère qu'on y retrouve habituellement. Et ce conte peut être vu aussi comme une légende d'origine de l'igname !

J'ai découvert cet ouvrage avec mon fils âgé de cinq ans et un de ses «tontons » kanak, dans le fond de la vallée de Po à Ponérihouen, lors de ma dernière mission. Nous avons tous été saisis par la pertinence des propos et des illustrations. L'auteur, jeune femme calédonienne qui a grandi à Koumac (nord-ouest de la Grande Terre) jusqu'à l'âge de vingt ans, a bien perçu les réalités de la vie quotidienne kanak dont elle a su si justement s'inspirer pour ce conte merveilleux, « fruit de [son] imagination $»$.

L'ouvrage commence par l'explication de seize mots locaux - bougna, cagou, cordyline, creek, gaïac, jamelonier, kaori, manou, mwata, nautou (qu'on écrit plutôt notou), niaouli, picot, pilou, plante à brûlure, popinée, toutoute - afin de permettre la compréhension du récit par tout lecteur, qu'il connaisse ou non la Nouvelle-Calédonie. Puis vient l'histoire. Le héros est un jeune garçon appelé Chane qui vit heureux, dans un pays magnifique, avec sa sœur cadette Sana et sa mère Ouvione-La-Courageuse. On l'appelle ainsi car elle est seule à prendre soin de sa famille, sans mari et sans père pour ses enfants. Et elle peut en être fière, nous dit-on, car ses taros, ses maniocs et ses patates sont les meilleurs de tous les environs. Comme nombre de femmes kanak, « elle s'appliquait à soigner sans relâche chaque plante, chaque arbuste » (p. 5). Et c'est là qu'apparaît la fleur d'igname à laquelle elle porte un attachement tout particulier : «On cultivait la fleur d'igname car on disait qu'elle réjouissait le cœur des bons esprits. Mais rares étaient ceux qui s'y essayaient. 